\title{
Impact of a sports and rehabilitation program on perception of quality of life in people with visual impairments
}

\author{
DOI: https://doi.org/10.5114/pq.2018.79742
}

\author{
Mariia Roztorhui, Alina Perederiy, Yuriy Briskin, Olexandr Tovstonoh, Khrystyna Khimenes, Valeryi Melnyk
}

Lviv State University of Physical Culture, Lviv, Ukraine

\section{Abstract}

Introduction. The aim of the research was to determine the influence degree of a sports and rehabilitation program on perception of quality of life among people with visual impairments.

Methods. Theoretical analysis, sociological methods (SF-36, 36-item Short Form Health Survey), pedagogical experiment, mathematical and statistical methods were applied. The research involved 34 people with visual impairments, who were divided into 3 groups, depending on the level of their visual impairment.

Results. It has been figured out that the perception of quality of life in people with visual impairments depends on the level of their vision loss. Among the blind, the perception is significantly lower $(p<0.05)$ than with moderate or severe visual impairment. A comparison of the evaluation results (with the SF-36 questionnaire) before and after the experiment allowed to determine the impact of the authors' program on the perception of quality of life in people with visual impairment.

Conclusions. A sports and rehabilitation program for people with visual impairments has been created. The program stipulates the fulfilment of rehabilitation tasks as well as tasks of sports training. It preconditions intensive sports training in the future. The implementation of the sports and rehabilitation program has been proved effective $(p<0.05)$ for the perception of quality of life in people with visual impairments.

Key words: blind, adaptive sport, physical health, mental health

\section{Introduction}

Nowadays, social sports awareness is being raised. With regard to athletes with disabilities, this fact is significant in the way that it results in shifting priorities from rehabilitation services application to sports training programs elaboration $[1,2]$. Reduction of the rehabilitation component (i.e. application of a system for athletes' adaptive sports training, which is popular nowadays) limits the sporting activity as well as the possibility to develop sports potential for people with disabilities when they try to achieve the highest result.

In terms of adaptive sports popularization, specialists have only dealt with sports rehabilitation within the framework of solving tasks aimed to restore the functions of the organism after sustaining sports injuries [3-5]. This fact is supposed to be the consequence of sports rehabilitation to be considered as a part (but not an individual stage) of the structure of long-term multiannual preparation of athletes with disabilities. Meanwhile, the specificity in achieving a sporting result (which is the characteristic feature for people with disabilities to integrate into society) depends on the nosological determinancy of the sport as well as the level of athletes' motor skills development for doing adaptive sports successfully [6-9]. Therefore, in the whole system of adaptive sports training, sports rehabilitation should be considered as an individual stage in the structure of long-term multiannual preparation of an athlete with disabilities [10]. This statement has also been substantiated by scientists who elucidate the importance of the individual stage, where the sports and rehabilitation program is implemented as well as the particular tasks are accomplished. These tasks are: to decrease the duration but to improve the effectiveness of the initial training; to develop motor skills and abilities; to improve the level of physical development and physical preparation; to promote formation of adaptive and compensatory mechanisms in the body of athletes with disabilities; to promote normalization of motor activity; to restore muscular strength; to prevent the development of muscle atrophy; to prevent and to treat contractures; to develop the ability to walk independently [11].

Solving the tasks of adaptive sports practice will provide conditions for self-realization and social integration that will enhance self-perception of quality of life for people with disabilities. As the compensation or reduction of the impact of lost functions are a guarantee of improving the self-perception of quality of life in people with visual impairments [12,13], it is necessary to determine the effectiveness of a sports and rehabilitation program in the process of their formation.

The purpose of the research was to determine the influence degree of a sports and rehabilitation program on the perception of quality of life among people with visual impairments.

\section{Subjects and methods}

\section{Subjects}

The research was conducted in Lviv and Rivne Invasport (Regional Center of Physical Culture and Sports for People with Disabilities). The subjects were informed about the purpose of the experiment, as well as they gave permission to disclose information about their participation. Medical information on visual acuity, eyesight, and peculiarities of the disease was provided by the Medical and Social Expert

Correspondence address: Mariia Roztorhui, Department of Theory of Sport and Physical Culture, Lviv State University of Physical Culture, 11 Kostiushko Str., Lviv, Ukraine, e-mail: mariia.roztorhyi@gmail.com 
Commission of Ukraine and received from the medical records examination. The experiment involved 34 people with visual impairments: 16 women and 18 men. The participants were divided into 3 groups, depending on the level of vision loss: blind, B1 (average age, $25.63 \pm 6.35$ years; $n=8$ ); persons with severe visual impairment, B2 (average age, $27.4 \pm$ 5.74 years; $n=10$ ); and those with moderate visual impairment, B3 (average age, $26.25 \pm 7.02$ years; $n=16$ ) with regard to the Classification Code of International Paralympic Committee [14]. Before the experiment, the participants did not specialize in sports sections. Among the subjects, 23 had visual impairments since birth and 11 acquired them during their lives ( 5 during childhood, 3 in adolescence, and 3 in adult age). The acquired visual impairments among the researched persons lasted 8-28 years.

\section{Process of the research}

The structure of the sports and rehabilitation program for people with visual impairments includes theoretical and practical parts (as illustrated in Figure 1). The theoretical part predetermines the acquisition of knowledge (on the problems of adaptive sports) as well as the development of mental processes and personality traits. The theoretical part is aimed to create optimal preparedness for social integration and competitive activities. In the whole sports and rehabilitation program for people with visual impairments, the theoretical part amounts $13.33 \%$ : psychological training accounts for $6.25 \%$ and theoretical preparedness for $7.08 \%$.
The practical part of the sports and rehabilitation program for people with visual impairments includes the following components: exercises for development of physical qualities; exercises to study the technology of various sports; recreational competitions; exercises to walk independently; self-grooming and self-service tips. The value of the practical part in the structure of the sports and rehabilitation program is $86.67 \%$. In order to develop of physical qualities, the following exercises are recommended: strength exercises, endurance exercises, velocity exercises, flexibility exercises, as well as exercises for the development of coordination qualities. In the whole structure of the program for people with visual impairments, the value of physical preparedness improvement accounts for $38.75 \%$. Athletics, swimming, strength sports, and football are included in the sports and rehabilitation program for people with visual impairments so as to study the techniques of various sports. In the structure of the program, there are $22.29 \%$ devoted to the study of various sports techniques.

The exercises which are helpful in adapting to daily life situations and self-service are supplemented by those used to master the technique of hand-tracking on the wall, footprints, self-defence technique (with the hand), as well as with the exercises applied to improve the skills of walking (with the help of another person) and the tactics of walking with tactile canes. The percentage of the above exercises amounts $15.62 \%$ in the structure of the sports and rehabilitation program. There are $5.00 \%$ assigned to recreational competitions and control classes.

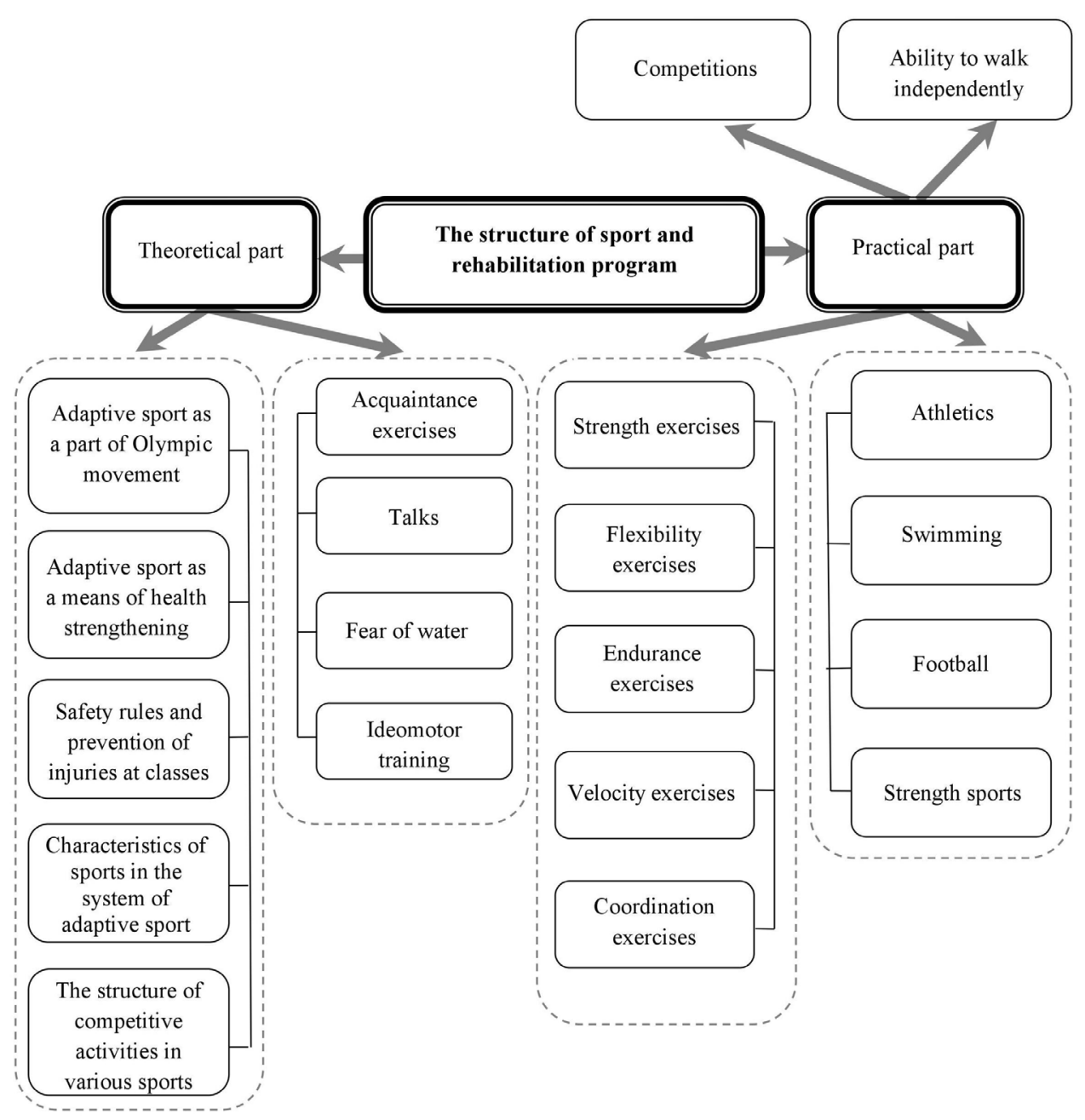

Figure 1. Structure of the sports and rehabilitation program for people with visual impairments 
It was difficult to transport people with visual impairments to the places of training because a large number of the experiment participants were limited in self-locomotion during the implementation of the sports and rehabilitation program. After performing the part of the program which was devoted to orientation and mobility training, most of the difficulties with active mobility of people with visual impairments were solved. Equal opportunities to participate in all tasks of the practical and theoretical part were ensured to all subjects. It was due to the distribution of participants to groups in accordance with the level of vision loss in the process of implementing the sports and rehabilitation program.

\section{Experimental protocol}

An adapted version of the MOS 36-item Short Form Health Survey (SF-36 questionnaire) in Ukrainian was used to assess the perception of quality of life among the people with visual impairments. The questionnaire was previously applied by Feshchenko et al. [15]. For testing blind people (group B1), a playback sound of SF-36 questions and answers are used. The questionnaire was applied to indicate the General physical health component and the General mental health component [16-18]. The General physical health component comprises the following scales: Physical functioning (11 questions), Role limitations due to physical health (4 questions), Bodily pain (2 questions), General health perceptions (5 questions). The General mental health component consists of such scales as: Vitality (4 questions), Social functioning (2 questions), Role limitations due to emotional problems (3 questions), Mental health (5 questions). Each of the scale indicators ranges from 0 to 100 points.

The SF-36 survey was conducted before and after the experiment. In order to implement the sports and rehabilitation program, the participants had to attend training sessions (40 hours) within the period of 1.5 months. They trained 3 times per week during 120 minutes.

\section{Statistical analysis}

The results were analysed with the Statistics for Windows Software (version 6.00). Previously, all variables were ana- lysed with the purpose to reveal the normality with the help of the Shapiro-Wilk test. The Mann-Whitney U test was applied to assess the significance of statistical differences in the results of the survey on the perception of quality of life among people with visual impairments of different groups. The sign test was used to compare the particular parameters between the groups before and after the experiment. The value of $p<0.05$ was considered statistically significant.

\section{Ethical approval}

The research related to human use has been complied with all the relevant national regulations and institutional policies, has followed the tenets of the Declaration of Helsinki, and has been approved by the authors' institutional review board or an equivalent committee.

\section{Informed consent}

Informed consent has been obtained from all individuals included in this study.

\section{Results}

In order to determine the effectiveness of the program implementation, the assessment of the perception of quality of life among people with visual impairments before the experiment (Table 1) and after the experiment (Table 2) was performed.

Having analysed the assessment of the perception of quality of life among people with visual impairments (with the SF-36) before the experiment, we detected differences in the General physical health component and General mental health component (depending on the level of vision loss). The indicators of the perception of quality of life among participants in group B1 (Physical functioning, General health, and Vitality scales) were statistically different: the level of reliability equalled $p<0.01$. Three scales (Role limitations - emotional, Mental health, and Role limitations - physical) were revealed to present differences between the B1 group and the B2 and B3 groups (the level of reliability, $p<0.05$ ). As for the Bodily pain and Social functioning scales, the indicators for participants with different levels of vision loss did not differ statistically significantly. No significant differences

Table 1. Perception of quality of life in people with visual impairments before the experiment

\begin{tabular}{|l|c|c|c|c|c|c|}
\hline Scale & $\begin{array}{c}\text { Group B1 } \\
(n=8) \\
\bar{x} \pm S D\end{array}$ & $\begin{array}{c}\text { Group B2 } \\
(n=10) \\
\bar{x} \pm S D\end{array}$ & $\begin{array}{c}\text { Group B3 } \\
(n=16) \\
\bar{x} \pm S D\end{array}$ & UB1-B2 & UB2-B3 & UB1-B3 \\
\hline Physical functioning & $57.50 \pm 5.35$ & $68.00 \pm 8.56$ & $70.94 \pm 10.68$ & $12^{*}$ & 59.5 & $21^{*}$ \\
\hline Role limitations - physical & $40.63 \pm 12.94$ & $57.50 \pm 12.08$ & $57.81 \pm 11.97$ & $17.5^{* *}$ & 79 & $27.5^{* *}$ \\
\hline Bodily pain & $57.50 \pm 10.18$ & $63.00 \pm 10.38$ & $63.63 \pm 13.11$ & 28 & 76.5 & 44 \\
\hline General health & $44.38 \pm 8.12$ & $54.60 \pm 7.46$ & $54.88 \pm 5.07$ & $13^{*}$ & 75 & $16^{*}$ \\
\hline Vitality & $44.38 \pm 4.96$ & $53.50 \pm 5.80$ & $53.75 \pm 4.65$ & $8^{*}$ & 75 & $10^{*}$ \\
\hline Social functioning & $60.94 \pm 14.07$ & $66.25 \pm 10.29$ & $67.97 \pm 17.66$ & 31.5 & 79.5 & 51.5 \\
\hline Role limitations - emotional & $37.50 \pm 11.79$ & $56.67 \pm 16.10$ & $58.33 \pm 19.25$ & $17^{* *}$ & 77.5 & $27.5^{\star *}$ \\
\hline Mental health & $56.00 \pm 8.28$ & $64.80 \pm 9.00$ & $65.50 \pm 6.51$ & $10^{* *}$ & 75 & $25^{* *}$ \\
\hline General physical health component & $39.61 \pm 1.45$ & $43.49 \pm 2.51$ & $43.74 \pm 2.95$ & $7^{*}$ & 72 & $9^{*}$ \\
\hline General mental health component & $39.71 \pm 3.03$ & $44.30 \pm 3.21$ & $44.60 \pm 3.84$ & $12^{*}$ & 78 & $12^{*}$ \\
\hline
\end{tabular}

* statistically significant results $(p<0.01),{ }^{* *}$ statistically significant results $(p<0.05)$

B1 - blind, B2 - severe visual impairment, B3 - moderate visual impairment, $\bar{x}$ - mean, $S D$ - standard deviation,

$U$ - Mann-Whitney U test 
Table 2. Perception of quality of life in people with visual impairments after the experiment

\begin{tabular}{|l|c|c|c|c|c|c|}
\hline Scale & $\begin{array}{c}\text { Group B1 } \\
(n=8) \\
\bar{x} \pm S D\end{array}$ & $\begin{array}{c}\text { Group B2 } \\
(n=10) \\
\bar{x} \pm S D\end{array}$ & $\begin{array}{c}\text { Group B3 } \\
(n=16) \\
\bar{x} \pm S D\end{array}$ & UB1-B2 & UB2-B3 & UB1-B3 \\
\hline Physical functioning & $73.13 \pm 7.04$ & $79.50 \pm 4.38$ & $80.63 \pm 3.59$ & $18^{* *}$ & 70.5 & $23.5^{*}$ \\
\hline Role limitations - physical & $59.38 \pm 12.94$ & $72.50 \pm 7.91$ & $70.31 \pm 10.08$ & $19^{* *}$ & 73 & $36^{* *}$ \\
\hline Bodily pain & $70.00 \pm 11.06$ & $71.60 \pm 6.92$ & $71.75 \pm 12.11$ & 38 & 77 & 63 \\
\hline General health & $58.25 \pm 9.16$ & $61.00 \pm 5.16$ & $61.06 \pm 5.23$ & 32.5 & 78 & 52.5 \\
\hline Vitality & $50.63 \pm 1.77$ & $58.00 \pm 3.50$ & $57.81 \pm 3.15$ & $2.5^{*}$ & 78.5 & $44^{*}$ \\
\hline Social functioning & $73.44 \pm 10.43$ & $75.00 \pm 5.89$ & $78.13 \pm 10.70$ & 39.5 & 70 & 55 \\
\hline Role limitations - emotional & $70.83 \pm 11.79$ & $80.00 \pm 17.21$ & $75.00 \pm 14.91$ & 29 & 68 & 56 \\
\hline Mental health & $64.00 \pm 5.66$ & $69.60 \pm 8.04$ & $69.25 \pm 6.32$ & 27 & 79 & 40 \\
\hline General physical health component & $45.71 \pm 3.53$ & $47.09 \pm 2.30$ & $47.29 \pm 3.12$ & 28 & 73 & 45 \\
\hline General mental health component & $45.58 \pm 1.06$ & $48.20 \pm 3.87$ & $47.67 \pm 2.63$ & 23 & 73 & $33^{* *}$ \\
\hline
\end{tabular}

* statistically significant results $(p<0.01),{ }^{* *}$ statistically significant results $(p<0.05)$

B1 - blind, B2 - severe visual impairment, B3 - moderate visual impairment, $\bar{x}$ - mean, $S D$ - standard deviation,

$U$ - Mann-Whitney U test

were detected in the perception of quality of life among people with different periods of visual impairments.

The second assessment, after fulfilling the sports and rehabilitation program, brought into light an improvement in the results of the perception of quality of life among people with visual impairments. Positive dynamics was revealed in the main two scales (General physical health component and General mental health component), as well as in the indicators for all other scales in the SF-36 questionnaire (Table 2).

After the experiment, a difference in the perception of quality of life indicators among people with different levels of vision loss was found out in the following scales: Physical functioning, Role limitations - physical, and Vitality. In the other scales, no significant difference has observed between the results of group B1 and groups B2 and B3. The obtained results signal the positive influence of the sports and rehabilitation program on the perception of quality of life in people with visual impairments. The most significant changes in the General physical health component and the General mental health component, as well as in some other scales were determined in group B1 (Table 3).

Positive changes in the Physical functioning scale, which were found by comparing the results before and after the experiment, indicate an increase in the level of adaptation in everyday life and self-service among people with visual impairments The most pronounced changes in the indicators on the Physical functioning scale after the implementation of the sports and rehabilitation program were observed in participants of group B1. This is obviously due to the lack of skills in using mobility tools and the low level of adaptation to daily life situation before the experiment in most representatives of this group. The increase in the indicators was $27.17 \%$ in group B1, $16.91 \%$ in group B2, and $13.66 \%$ in group B3 (Table 3).

As a result of the sports and rehabilitation program implementation, a significant increase of different scales indicators was revealed among all experiment participants.

Table 3. Indicators of percentage increase in the perception of quality of life in people with visual impairments (as a result of the sports and rehabilitation program implementation)

\begin{tabular}{|l|c|c|c|c|c|c|}
\hline \multirow{2}{*}{ Scale } & \multicolumn{2}{|c|}{ Group B1 $(n=8)$} & \multicolumn{2}{|c|}{ Group B2 $(n=10)$} & \multicolumn{2}{c|}{ Group B3 $(n=16)$} \\
\cline { 2 - 7 } & $\%$ & $p$ & $\%$ & $p$ & $\%$ & $p$ \\
\hline Physical functioning & 27.17 & $<0.008$ & 16.91 & $<0.008$ & 13.66 & $<0.003$ \\
\hline Role limitations - physical & 46.15 & $<0.031$ & 26.09 & $<0.031$ & 21.62 & $<0.008$ \\
\hline Bodily pain & 21.74 & $<0.031$ & 13.65 & $<0.031$ & 12.77 & $<0.008$ \\
\hline General health & 31.27 & $<0.008$ & 11.72 & $<0.008$ & 11.28 & $<0.001$ \\
\hline Vitality & 14.08 & $<0.031$ & 8.41 & $<0.031$ & 7.56 & $<0.004$ \\
\hline Social functioning & 20.51 & $<0.031$ & 13.21 & $<0.031$ & 14.94 & $<0.012$ \\
\hline Role limitations- emotional & 88.89 & $<0.008$ & 41.18 & $<0.031$ & 28.57 & $<0.021$ \\
\hline Mental health & 14.29 & $<0.016$ & 7.41 & $<0.002$ & 5.73 & $<0.001$ \\
\hline General physical health component & 15.39 & $<0.008$ & 8.92 & $<0.021$ & 8.11 & $<0.021$ \\
\hline General mental health component & 14.77 & $<0.008$ & 8.81 & $<0.021$ & 6.88 & $<0.021$ \\
\hline
\end{tabular}




\section{Discussion}

The analysis of the research results (obtained with the SF-36 questionnaire) proved that the level of visual impairment had a significant impact on the perception of quality of life among the people with visual impairments. The indicators of the scales of General physical health component and General mental health component ranged from 39.61 to 44.30 in all of the participants of the experiment and thus they demonstrated an unsatisfactory self-perception of quality of life.

With regard to the particular indicators, one should mention groups B2 and B3, with their indicators reaching up to 50 points. Pavlova et al. [19] claim that the indicators of perception of quality of life which do not exceed 50 points can be considered as unsatisfactory. Furthermore, they assert that chronic diseases and disorders cause obstacles for effective physical activity, thus leading to a reduction in the SF36 questionnaire results. In the presented research, the perception of quality of life indicators (regarding Role limitations - physical, General health, Vitality, and Role limitations - emotional) in the B1 group ranged from 37.50 to 44.38 before the experiment. The statement about a significant influence of the level of vision loss on the perception of quality of life was confirmed in the studies by Chia et al. [20], Masaki [21], and Dev et al. [22], who compared the perception of quality of life among people with severe eye lesions, blind people, and healthy ones.

The effectiveness of the sports and rehabilitation program was determined in the analyses of the perception of quality of life among people with visual impairments after the experiment. The positive impact of the sports and rehabilitation program on the perception of quality of life was recognized as an important result of the research. The perceived quality of life among people with visual impairments increased in such scales as Physical functioning, Role limitations - physical, Bodily pain, General health. The role of these scales is essential in the way they form the general component of physical health. For example, the percentage increase in the perception of quality of life for these scales in group B1 was $27.17 \%, 46.15 \%, 21.74 \%$, and $31.27 \%$, respectively. This fact signals the benefits of creating adaptive sports mechanisms that will allow to compensate or reduce the impact of lost functions on the perception of quality of life in people with disability [7, 23-25].

The changes as a result of the experiment (concerning Vitality, Social functioning, Role limitations - emotional, and Mental health, which are the parts of the General mental health component) turned out the most pronounced in the B1 group. People with visual impairments are inclined to inadequately assess their mental health and social activity [22]. This fact makes it clear why they are fully satisfied with their social activity and emotional state. We came to this statement after analysing the responses of the participants to the questions about their perception of quality of life in terms of the Role limitations - emotional scale. The results of this scale among people with visual impairments grew up to $88.89 \%$ (group B1), 41.18\% (group B2), and 28.57\% (group B3) after the implementation of the sports and rehabilitation program. The indicators of this scale were revealed to be very similar to those among healthy people [19]. This result, obtained after the experiment, indicates a significant influence of sports on the emotional state and the daily activity of people with visual impairments.

\section{Limitations}

The research has some limitations. Firstly, the number of the participants in the experiment was not large but limited to those from the Lviv and Rivne regions of Ukraine. This limitation negatively affects any extrapolation of the results to all people with disabilities. Secondly, a mention should be made that the age of the participants ranged from 16 to 43 years; thus, the results of the experiment may not be effective for other age groups. Thirdly, it should be taken into account that the assessment of the perceived quality of life among people with visual impairments was performed with the SF-36 questionnaire, in which individual characteristics could not be identified.

\section{Conclusions}

The analysis of the results, obtained with the SF-36 questionnaire, elucidates the fact of a correlation between the level of visual impairment and the perception of quality of life among people with visual impairments. A positive dynamics was observed of the indicators of the General physical health component and the General mental health component; also, an increase of the level of preserved vision was revealed. Within the research, a sports and rehabilitation program of training for visually impaired persons was developed. It includes theoretical and practical parts. The main task of the former is to increase the theoretical awareness and psychological health of visually impaired people. The practical part is aimed at increasing the level of physical preparedness; mastering the techniques of various sports; developing skills of walking independently, and providing tips for self-grooming.

The experimental testing of the sports and rehabilitation program has confirmed a significant increase in the indicators of the perceived quality of life in people with visual impairments. It has been observed that the blind demonstrate the greatest changes in the indicators of the perception of quality of life when involved in the sports and rehabilitation program. This shows that the positive impact of the sports and rehabilitation program among the blind is significantly higher $(p<0.01)$ than among people with moderate and severe visual impairments.

\section{Disclosure statement}

No author has any financial interest or received any financial benefit from this research.

\section{Conflict of interest}

The authors state no conflict of interest.

\section{References}

1. Dehghansai N, Lemez S, Wattie N, Baker J. A systematic review of influences on development of athletes with disabilities. Adapt Phys Activ Q. 2017;34(1):72-90; doi: 10.1123/APAQ.2016-0030.

2. McNamee M. Paralympism, paralympic values and disability sport: a conceptual and ethical critique. Disabil Rehabil. 2017;39(2):201-209; doi: 10.3109/09638288. 2015.1095247.

3. Fagher K, Jacobsson J, Timpka T, Dahlström Ö, Lexell J. The sports-related injuries and illnesses in Paralympic Sport Study (SRIIPSS): a study protocol for a prospective longitudinal study. BMC Sports Sci Med Rehabil. 2016; 8(1):28; doi: 10.1186/s13102-016-0053-x. Available from: https://www.ncbi.nlm.nih.gov/pmc/articles/PMC5004301/ pdf/13102_2016_Article_53.pdf. 
4. Willick SE, Cushman DM, Blauwet CA, Emery C, Webborn N, Derman W, et al. The epidemiology of injuries in powerlifting at the London 2012 Paralympic Games: an analysis of 1411 athlete-days. Scand J Med Sci Sports. 2016;26(10):1233-1238; doi: 10.1111/sms.12554.

5. Blauwet C, Willick SE. The Paralympic Movement: using sports to promote health, disability rights, and social integration for athletes with disabilities. PM\&R. 2012;4(11): 851-856; doi: 10.1016/j.pmrj.2012.08.015.

6. Weiler R, Van Mechelen W, Fuller C, Verhagen E. Sport injuries sustained by athletes with disability: a systematic review. Sports Med. 2016;46(8):1141-1153; doi: 10.1007/ s40279-016-0478-0.

7. Groff DG, Lundberg NR, Zabriskie RB. Influence of adapted sport on quality of life: perceptions of athletes with cerebral palsy. Disabil Rehabil. 2009;31(4):318-326; doi: 10.1080/09638280801976233.

8. Lastuka A, Cottingham M. The effect of adaptive sports on employment among people with disabilities. Disabil Rehabil. 2015;38(8):742-748; doi: 10.3109/09638288. 2015.1059497.

9. Sobiecka J. Sports activity as a form of increasing efficiency in rehabilitation of handicapped people [in Polish]. Wiad Lek. 2002;55(Suppl. 1):454-458.

10. Jaarsma EA, Dekker R, Geertzen JH, Dijkstra PU. Sports participation after rehabilitation: barriers and facilitators. JRehabilMed.2016;48(1):72-79; doi:10.2340/165019772017.

11. Lynets M, Perederiy A, Strokatov V, Briskin Y. Correction and rehabilitation program for athletes with disability as an objective prerequisite for sport preparation [in Russian]. Nauka v Olimpijskom Sporte. 2002;2:92-94.

12. Scott IU, Smiddy WE, Schiffman J, Feuer WJ, Pappas CJ. Quality of life of low-vision patients and the impact of lowvision services. Am J Ophthalmol. 1999;128(1):54-62; doi: 10.1016/S0002-9394(99)00108-7.

13. Khil L, Wellmann J, Berger K. Impact of combined sensory impairments on health-related quality of life. Qual Life Res. 2015;24(9):2099-2103; doi: 10.1007/s11136015-0941-7.

14. Mann DL, Ravensbergen HJC. International Paralympic Committee (IPC) and International Blind Sports Federation (IBSA) joint position stand on the sport-specific classification of athletes with vision impairment. Sports Med. 2018; doi: 10.1007/s40279-18-0949-6. Available from: https://link.springer.com/content/pdf/10.1007\%2 Fs40279-018-0949-6.pdf.

15. Feshchenko Y, Mostovoy Y, Babiychuk Y. The procedure of adaptation of international quality of life questionnaire MOS SF-36 in Ukraine. The experience of administration in asthma patients [in Ukrainian]. Ukrainian Pulmonology Journal. 2002;3:9-11.

16. Ware JE Jr, Sherbourne CD. The MOS 36-item shortform health survey (SF-36): I. Conceptual framework and item selection. Med Care. 1992;30(6):473-483.

17. Hays RD, Shapiro MF. An overview of generic healthrelated quality of life measures for HIV research. Qual Life Res. 1992;1(2):91-97; doi: 10.1007/BF00439716.

18. Ware JE Jr, Keller SD, Gandek B, Brazier JE, Sullivan M. Evaluating translations of health status questionnaires. Methods from the IQOLA project. International Quality of Life Assessment. Int J Technol Assess Health Care. 1995;11(3):525-551; doi: 10.1017/S0266462300008710.

19. Pavlova I, Vynogradskyi B, Ripak I, Zikrach D, Borek Z. Prognostication of health-related life quality of Ukrainian residents due to physical activity level. J Phys Educ Sport. 2016;16(2):418-423; doi: 10.7752/jpes.2016.02065.

20. Chia EM, Wang JJ, Rochtchina E, Smith W, Cumming RR, Mitchell P. Impact of bilateral visual impairment on healthrelated quality of life: the Blue Mountains Eye Study. Invest Ophthalmol Vis Sci. 2004;45(1):71-76; doi: 10.1167/ iovs.03-0661.

21. Masaki I. Reduced health-related quality of life among Japanese college students with visual impairment. Biopsychosoc Med. 2015;9:18; doi: 10.1186/s13030-0150045-1. Available from: https://bpsmedicine.biomedcentral.com/track/pdf/10.1186/s13030-015-0045-1.

22. Dev MK, Paudel N, Joshi ND, Shah DN, Subba S. Psychosocial impact of visual impairment on health-related quality of life among nursing home residents. BMC Health Serv Res. 2014;14:345; doi: 10.1186/1472-6963-14-345. Available from: https://www.ncbi.nlm.nih.gov/pmc/articles/PMC4138377/pdf/12913_2014_Article_3447.pdf.

23. Houwen S, Visscher C, Hartman E, Lemmink KA. Gross motor skills and sports participation of children with visual impairments. Res Q Exerc Sport. 2007;78(2):16-23; doi: 10.1080/02701367.2007.10762235.

24. Sahlin KB, Lexell J. Impact of organized sports on activity, participation, and quality of life in people with neurologic disabilities. PM\&R. 2015;7(10):1081-1088; doi: 10.1016/j.pmrj.2015.03.019.

25. Yazicioglu K, Yavuz F, Goktepe AS, Tan AK. Influence of adapted sports on quality of life and life satisfaction in sport participants and non-sport participants with physical disabilities. Disabil Health J. 2012;5(4):249-253; doi: 10.1016/j.dhjo.2012.05.003. 